Virginie H. van Waning

Robin M. F. van der Weiden

Peter W. de Feiter

Arjen J. B. W. Brouwers

\section{Cardiac arrest in pregnancy: lessons to be learned!}

Accepted: 7 December 2011

Published online: 10 February 2012

(C) Copyright jointly held by Springer and ESICM 2012

Dear Editor,

Cardiac arrest in pregnancy is a rare event with an incidence of 1 per 30,000 pregnancies in western countries. However, it is important for emergency physicians to be familiar with the indications for performing a perimortem caesarean section (PMCS). We report two cases of PMCS performed during cardiopulmonary resuscitation (CPR).

The first case, a 37-year-old female, G1P0, had a medical history of epilepsy. Her pregnancy was complicated at 30 weeks gestation by a single uncomplicated grand mal seizure as a result of low valproate level. She was admitted, at 35 weeks gestation, for pregnancy-induced hypertension. Laboratory results were normal. Two days after admission she was found unresponsive. She had pulseless electrical activity (PEA) and hypoxia due to an epileptic insult was considered the diagnosis. She was treated according to the advanced cardiac life support protocol. Fifteen min after the start of CPR a PMCS was performed. There was an immediate return of spontaneous circulation (ROSC). Unfortunately 2 days later her treatment had to be withdrawn, because of poor neurological prognosis. The male infant (2,300 g; Apgar score 0/6/7) had seizures and was resuscitated. The baby survived, and follow-up 12 months after delivery showed normal growth and slight neurologic development impairment.

The second case, a 35-year-old female, G3P1, had an uneventful medical history and was admitted for induction of labour at 41 weeks and 3 days gestation. After spontaneous rupture of membranes the patient suffered from dyspnoea, hypotension, bradycardia, and cyanosis. Supplemental oxygen and left lateral tilt position did not alleviate the symptoms. Upon arrival of the resuscitation team the patient had PEA. Amniotic fluid embolism was considered the diagnosis. Perimortem caesarean section was performed in the delivery room after $4 \mathrm{~min}$, resulting in the immediate ROSC and the birth of a girl $(3,450 \mathrm{~g}$; Apgar score $2 / 6 / 7$ ). Both were discharged without abnormalities.

During CPR in late pregnancy the patient should be positioned in left lateral tilt to alleviate aortocaval compression [1]. If sufficient cardiac output is not achieved within $4 \mathrm{~min}$ after cardiac arrest, in a pregnancy of more than 20 weeks gestation, PMCS must be considered [1].

In our cases an important observation was made that immediately after delivery of the infant there was a ROSC, indicating that indeed pooling of blood in the uterus and placenta as well as aortocaval compression played a pivotal role in the prior unsuccessful resuscitation.

We performed two PMCS in our hospital. The first PMCS was before the courses based on the Managing Obstetric Emergencies and Trauma (MOET) principles were given at our institution [2,3]. Important differences between the first and second case were the immediate awareness of the necessity to perform PMCS in the first 4 min after persistent cardiopulmonary arrest, the availability of an emergency PMCS box [4] in the delivery room and the notion to perform the PMCS on the spot. This all resulted in a much faster delivery of the baby and more importantly much faster return of the maternal circulation, resulting in favourable clinical outcome for both mother and child.

\section{References}

1. Soar J, Perkins GD, Abbas G, Alfonzo A, Barelli A, Bierens JJ, Brugger H, Deakin CD, Dunning J, Georgiou M, Handley AJ, Lockey DJ, Paal P, Sandroni C, Thies KC, Zideman DA, Nolan JP (2010) European Resuscitation Council Guidelines for Resuscitation 2010 Section 8. Cardiac arrest in special circumstances: electrolyte abnormalities, poisoning, drowning, accidental hypothermia, hyperthermia, asthma, anaphylaxis, cardiac surgery, trauma, pregnancy, electrocution. Resuscitation 81:1400-1433

2. Grady K, Howell C, Cox C (2009) MOET course manual. RCOG Press, London

3. Dijkman A, Huisman CM, Smit M, Schutte JM, Zwart JJ, van Roosmalen JJ, Oepkes D (2010) Cardiac arrest in pregnancy: increasing use of perimortem caesarean section due to emergency skills training? BJOG 117:282-287

4. Warraich Q, Esen U (2010) For perimortem caesarean section, the surgical knife is the most important instrument. BJOG 117:768

V. H. van Waning (๘)

A. J. B. W. Brouwers

Department of Internal Medicine, Intensive Care Unit, Sint Franciscus Gasthuis, 3045 PM Rotterdam,

The Netherlands

e-mail: v.vanwaning@sfg.nl

Tel.: +31-10-4616149

Fax: +31-10-4612692

R. M. F. van der Weiden

Department of Obstetrics and Gynaecology, Sint Franciscus Gasthuis,

3045 PM Rotterdam, The Netherlands

\section{P. W. de Feiter}

Department of Surgery, Intensive Care Unit, Sint Franciscus Gasthuis,

3045 PM Rotterdam, The Netherlands 